\title{
Minimization of a Quasi-linear Ginzburg-Landau type energy
}

\author{
Rejeb Hadiji* and Carmen Perugia ${ }^{\dagger}$
}

\begin{abstract}
Let $G$ be a smooth bounded domain in $\mathbb{R}^{2}$. Consider the functional

$$
E_{\varepsilon}(u)=\frac{1}{2} \int_{G}\left(p_{0}+t|x|^{k}|u|^{l}\right)|\nabla u|^{2}+\frac{1}{4 \varepsilon^{2}} \int_{G}\left(1-|u|^{2}\right)^{2}
$$

on the set $H_{g}^{1}(G, \mathbf{C})=\left\{u \in H^{1}(G, \mathbf{C}) ; u=g\right.$ on $\left.\partial G\right\}$ where $g$ is a given boundary data with degree $d \geq 0$. In this paper we will study the behaviour of minimizers $u_{\varepsilon}$ of $E_{\varepsilon}$ and we will estimate the energy $E_{\varepsilon}\left(u_{\varepsilon}\right)$.

Keywords: Ginzburg-Landau equation, Quasi-linear problem, $S^{1}$ valued map.

Mathemathics Subject Classification (2000) : 35B25, 35J55, 35B40.
\end{abstract}

\section{Introduction}

Let $G$ be a bounded smooth domain of $\mathbf{R}^{2}, g: \partial G \rightarrow S^{1}$ a smooth boundary data of degree $d \geq 0$. For $\varepsilon>0, p_{0}>0, t>0, k \geq 2$ and $l \geq 2$ define the following functional of Ginzburg -Landau type

$$
E_{\varepsilon}(u)=\frac{1}{2} \int_{G}\left(p_{0}+t|x|^{k}|u|^{l}\right)|\nabla u|^{2}+\frac{1}{4 \varepsilon^{2}} \int_{G}\left(1-|u|^{2}\right)^{2}
$$

on the set

$$
H_{g}^{1}(G, \mathbf{C})=\left\{u \in H^{1}(G, \mathbf{C}) ; u=g \text { on } \partial G\right\} .
$$

We shall understand that if $\int_{G}|u|^{l}|\nabla u|^{2}=\infty$ then $E_{\varepsilon}(u)=\infty$. In this paper we are interested in the study of the asymptotic behaviour of

$$
\min _{u \in H_{g}^{1}(G, \mathbf{C})} E_{\varepsilon}(u)
$$

*Université Paris-Est, Laboratoire d'Analyse et de Mathématiques Appliquées, CNRS UMR 8050, UFR des Sciences et Technologie, 61, Avenue du Général de Gaulle Bât. P3, 4e étage, 94010 Créteil Cedex, France. e-mail: hadiji@univ-paris12.fr

${ }^{\dagger}$ Universitá del Sannio, Dipartimento di Studi Geologici ed Ambientali, Via Dei Mulini 59/A Palazzo Inarcassa, 82100, Benevento, Italia. e-mail: perugia@unisannio.it 
When $t=0, k=0$ and $d=0$, Bethuel, Brezis and Hélein [BBH1] showed that as $\varepsilon$ tends to $0, u_{\varepsilon}$ tends to a harmonic $u_{0}$ which is equal to $g$ on $\partial G$ in $C^{1, \alpha}(\bar{G})$. It is easy to adapt the same method as in [BBH1] to obtain the same result when $k \neq 0$.

The case when $t=0$ and $d>0$, corresponding to the Ginzburg-Landau energy, was studied by Bethuel, Brezis and Hélein in [BBH2] (see also Struwe [S]), where it was shown that :

(i) for a subsequence $\varepsilon_{n} \rightarrow 0$ we have, $u_{\varepsilon_{n}} \rightarrow u_{*}=e^{i \phi} \prod_{j=1}^{d} \frac{z-a_{j}}{\left|z-a_{j}\right|}$ in $C^{1, \alpha}\left(\bar{G} \backslash\left\{a_{1}, \ldots, a_{d}\right\}\right)$, where $a_{1}, \ldots, a_{d}$ are distinct points in $G$ and $\phi$ is a smooth harmonic function determined by the requirement $u_{*}=g$ on $\partial G$.

(ii) $E_{\varepsilon}\left(u_{\varepsilon}\right)=2 \pi d|\log \varepsilon|+O(1)$ as $\varepsilon \rightarrow 0$.

In the case where $t>0$ and $l=0$, is studied the problem for more general weight depending only on $x$, see $[B H 1,2,3]$ and $[A S 1,2]$. They showed that the presence of the weight forces the location of the vortices near the minima of the weight and when the degree is greater than the number of the minima of $p$ the interaction between vortices led to a term of order $\ln \ln \frac{1}{\varepsilon}$. It is also showed in the above references that the zeroes of $u_{\varepsilon}$ are located, for small $\varepsilon$, near the minima of the weight.

In this paper, we study the effect of the presence of $|u|$ in the weight $p_{0}+t|x|^{k} s^{l}$. Our weight is a particular one and gives a significant situation. For instance, if we consider the case where $k=0$, we show that we obtain a similar results of convergence as in $[B B H 1]$ but the energy is greater than their energy. More precisely, in Theorem 1 we examine the case $\operatorname{deg}(g, \partial G)=0, k \geq 0$ and $l \geq 0$. In Theorem 2 we examine the case $\operatorname{deg}(g, \partial G)>0$, $k=0$ and $l \geq 0$. In Theorem 3 we examine the most general case $\operatorname{deg}(g, \partial G)>0, k \neq 0$ and $l \neq 0$. In both the last two cases we obtain a convergence result for a sequence of minimizers of our problem and we show that under a small perturbation of the weight $p_{0}+t|x|^{k} s^{l}$ the singularities of the limit problem are minima of $p_{0}+t|x|^{k}$. As regards the energy, in Theorem 2 , as $\varepsilon_{n} \rightarrow 0$ we get

$$
E_{\varepsilon_{n}}\left(u_{\varepsilon_{n}}\right)=\pi d\left(p_{0}+t\right) \ln \frac{1}{\varepsilon_{n}}+O(1)
$$

while in Theorem 3, where without loss of generality we can suppose that $0 \in G$, as $\varepsilon_{n} \rightarrow 0$ we obtain

$$
E_{\varepsilon_{n}}\left(u_{\varepsilon_{n}}\right)=\pi p_{0}\left(d \ln \frac{1}{\varepsilon_{n}}+\frac{d^{2}-d}{k} \ln \ln \frac{1}{\varepsilon_{n}}\right)+O(1) .
$$

The motivation of our study for the functional (1.1) comes from type II superconductors in the presence of vortices see $[\mathrm{AS} 1,2],[\mathrm{BH} 1,2,3]$, [DeG] and $[\mathrm{R}]$. 
The presence of the weight function is motivated by the problem of pinning of vortices. It forces the location of the vortices to some favorite sites. In the case where $l=0$ the regions where the weight is relatively small are called weak links see $[D G]$. So, we expect that the minima of the weight $p_{0}+t|x|^{k} s^{l}$ will play an important role. As we shall show below, the zeroes of a minimizer of our problem are located, for small $\varepsilon$, near the minima of $p_{0}+t|x|^{k}$.

\section{Setting of the problem and some preliminary results}

At first, let us recall a definition and a lemma contained in $[B]$.

Definition 1. Let $\Omega$ an open set of $\mathbb{R}^{p}, 1 \leq p \leq \infty$, ( $\left.\Omega, \mathfrak{I}, \mu\right)$ denote a measure space with $\mu$ non-negative and finite and $\mathfrak{I}$ is $\mu$-complete. Set $\mathbf{B}_{n}$ the borel $\sigma$-field of $\mathbb{R}^{n}$. A function $\left.\left.f: \Omega \times \mathbf{R}^{m} \times \mathbf{R}^{n} \rightarrow\right]-\infty,+\infty\right]$ is said to be a normal-convex integrand if $f$ is $\mathfrak{I} \otimes \mathbf{B}_{m} \otimes \mathbf{B}_{n}$-measurable function and there exists a $\mu$-negligible set $N \subset \Omega$ such that

$$
\begin{aligned}
& f(x, \cdot, \cdot) \text { is l.s.c. on } \mathbf{R}^{m} \times \mathbf{R}^{n} \text { for every } x \in \Omega-N \\
& f(x, s, \cdot) \text { is convex on } \mathbf{R}^{n} \text { for every } x \in \Omega-N, s \in \mathbf{R}^{m} .
\end{aligned}
$$

Lemma 2.1. Let $\Omega$ a bounded open set of $\mathbf{R}^{n}$ with Lipschitz boundary and let $f: \Omega \times$ $\mathbf{R}^{m} \times \mathbf{R}^{m n} \rightarrow[0,+\infty]$ be a normal-convex integrand in the sense of Definition 1. Then the functional

$$
F(u)=\int_{\Omega} f(x, u, \nabla u)
$$

is sequentially weakly $W^{1,1}\left(\Omega, \mathbf{R}^{m}\right)-$ l.s.c.

As a consequence of Lemma 2.1 we have

Lemma 2.2. Let $G$ be a bounded regular open set of $\mathbf{R}^{2}$. Then, the functionals

$$
F_{2}(u)=\int_{G}\left(p_{0}+t|x|^{k}|u|^{l}\right)|\nabla u|^{2}
$$

and

$$
F_{1}(u)=\int_{G}|x|^{k}|u|^{l}|\nabla u|^{2}
$$

are sequentially weakly $W^{1,1}\left(G, \mathbf{R}^{2}\right)$ - l.s.c.

Proof. For the first functional it is enough to apply Lemma 2.1 with $\Omega=G, m=n=2$ and $f(x, s, w)=\left(p_{0}+t|x|^{k}|s|^{l}\right)|w|^{2}$. About the latter one, it is enough to observe that it is the sum of two functionals sequentially weakly $W^{1,1}\left(G, \mathbf{R}^{2}\right)-$ l.s.c. 
Set

$$
p(x, s)=p_{0}+t|x|^{k}|s|^{l} .
$$

We have

Proposition 2.1. The infimum

$$
\inf _{u \in H_{g}^{1}(G, \mathbf{C})} E_{\varepsilon}(u)
$$

is achieved by some $u_{\varepsilon}$ which is smooth and satisfies

$$
\left\|u_{\varepsilon}\right\|_{L^{\infty}} \leq 1
$$

Moreover, $u_{\varepsilon}$ satisfies the Euler equation

$$
\begin{cases}-\operatorname{div}\left(p \nabla u_{\varepsilon}\right)+\frac{l t}{2}|x|^{k}\left|u_{\varepsilon}\right|^{l-2}\left|\nabla u_{\varepsilon}\right|^{2} u_{\varepsilon}=\frac{1}{\varepsilon^{2}}\left(1-\left|u_{\varepsilon}\right|^{2}\right) u_{\varepsilon} & \text { in } G \\ u_{\varepsilon}=g & \text { on } \partial G\end{cases}
$$

and that there exists $t_{0}=t_{0}\left(G, g, p_{0}, l, k\right)>0, \varepsilon_{0}=\varepsilon_{0}\left(G, g, p_{0}, l, k\right)>0$ such that

$$
\left\|\nabla u_{\varepsilon}\right\|_{\infty} \leq \frac{C}{\varepsilon} \text { for } t \leq t_{0} \text { and } \varepsilon \leq \varepsilon_{0}
$$

where $C$ is a constant independent of $\varepsilon$.

Proof. Using Lemma 2.2 we obtain that the infimum of $(2.5)$ is achieved by a function $u_{\varepsilon}$ Moreover, using Theorem 1.7 in $[K M]$ we obtain the regularity of any minimizer $u_{\varepsilon}$. Now, let us prove (2.6). Set $B=\left\{x \in G\right.$ s.t. $\left.\left|u_{\varepsilon}(x)\right|>1\right\}$. Suppose that $|B|>0$. Let us define $v_{\varepsilon} \in H^{1}\left(G, \mathbf{R}^{2}\right)$ by

$$
v_{\varepsilon}(x)= \begin{cases}u_{\varepsilon}(x) & \text { in } G \backslash B \\ \frac{u_{\varepsilon}(x)}{\left|u_{\varepsilon}(x)\right|} & \text { in } B\end{cases}
$$

We have

$$
\frac{1}{4 \varepsilon^{2}} \int_{G}\left(1-\left|v_{\varepsilon}\right|^{2}\right)^{2} \leq \frac{1}{4 \varepsilon^{2}} \int_{G}\left(1-\left|u_{\varepsilon}\right|^{2}\right)^{2}
$$

As $\left|v_{\varepsilon}\right|=1$ on $B$

$$
p_{0}+t|x|^{2}\left|v_{\varepsilon}\right|^{l}=p_{0}+t|x|^{k}<p_{0}+t|x|^{k}\left|u_{\varepsilon}\right|^{l} \text { on } B .
$$

It is easy to see that

$$
\left|\nabla u_{\varepsilon}\right|^{2}=\left|u_{\varepsilon}\right|^{2}\left|\nabla v_{\varepsilon}\right|^{2}+\left.|\nabla| u_{\varepsilon}\right|^{2}
$$

Therefore we have

$$
\left|\nabla v_{\varepsilon}\right|^{2} \leq\left|\nabla u_{\varepsilon}\right|^{2}
$$


By $(2.9),(2.10)$ and $(2.11)$ we get

$$
E_{\varepsilon}\left(v_{\varepsilon}\right)<E_{\varepsilon}\left(u_{\varepsilon}\right)
$$

but this is impossible since $u_{\varepsilon}$ is a minimizer. Then $|B|=0$ and consequently we obtain $\left|u_{\varepsilon}\right| \leq 1$ in $G$. It is easy to see that $u_{\varepsilon}$ satisfies the Euler equation (2.7).

Finally, in order to prove (2.8), we need the following result

$$
\left.\left.|\nabla| u\right|^{2}\right|^{2}=4|u \nabla u|^{2}
$$

Indeed, we observe that

$$
\nabla|u|^{2}=\left(2 u_{1} \partial_{x} u_{1}+2 u_{2} \partial_{x} u_{2} ; 2 u_{1} \partial_{y} u_{1}+2 u_{2} \partial_{y} u_{2}\right)
$$

then

$$
\begin{gathered}
\left.\left.|\nabla| u\right|^{2}\right|^{2}=\left(2 u_{1} \partial_{x} u_{1}+2 u_{2} \partial_{x} u_{2}\right)^{2}+\left(2 u_{1} \partial_{y} u_{1}+2 u_{2} \partial_{y} u_{2}\right)^{2} \\
=4 u_{1}^{2}\left(\partial_{x} u_{1}\right)^{2}+4 u_{2}^{2}\left(\partial_{x} u_{2}\right)^{2}++8 u_{1} u_{2} \partial_{x} u_{1} \partial_{x} u_{2} \\
+4 u_{1}^{2}\left(\partial_{y} u_{1}\right)^{2}+4 u_{2}^{2}\left(\partial_{y} u_{2}\right)^{2}+8 u_{1} u_{2} \partial_{x} u_{1} \partial_{x} u_{2} \\
=4\left(u_{1}^{2}\left|\nabla u_{1}\right|^{2}+2 u_{1} u_{2} \nabla u_{1} \cdot \nabla u_{2}+u_{2}^{2}\left|\nabla u_{2}\right|^{2}\right)=4|u \nabla u|^{2} .
\end{gathered}
$$

Now let us consider the equation (2.7) and have

$$
\begin{aligned}
-\Delta u_{\varepsilon}= & k t \frac{|x|^{k-2} x\left|u_{\varepsilon}\right|^{l}}{p_{0}+t|x|^{k}\left|u_{\varepsilon}\right|^{l}} \nabla u_{\varepsilon}+\frac{l}{2} t \frac{|x|^{k}\left|u_{\varepsilon}\right|^{l-2} \nabla\left|u_{\varepsilon}\right|^{2} \nabla u_{\varepsilon}}{p_{0}+t|x|^{k}\left|u_{\varepsilon}\right|^{l}} \\
& +\frac{1}{\varepsilon^{2}} \frac{\left(1-\left|u_{\varepsilon}\right|^{2}\right)}{p_{0}+t|x|^{k}\left|u_{\varepsilon}\right|^{l}} u_{\varepsilon}-\frac{l t}{2} \frac{|x|^{k}\left|u_{\varepsilon}\right|^{l-2}\left|\nabla u_{\varepsilon}\right|^{2} u_{\varepsilon}}{p_{0}+t|x|^{k}\left|u_{\varepsilon}\right|^{l}}
\end{aligned}
$$

and by (2.12) we obtain

$$
\left|\Delta u_{\varepsilon}\right| \leq k t \frac{|x|^{k-1}\left|u_{\varepsilon}\right|^{l}}{p_{0}+t|x|^{k}\left|u_{\varepsilon}\right|^{l}}\left|\nabla u_{\varepsilon}\right|+\frac{3}{2} l t \frac{|x|^{k}\left|u_{\varepsilon}\right|^{l-1}}{p_{0}+t|x|^{k}\left|u_{\varepsilon}\right|^{l}}\left|\nabla u_{\varepsilon}\right|^{2}+\frac{C}{\varepsilon^{2}} .
$$

Let $A$ be the diameter of $G$. Since $\left|u_{\varepsilon}\right| \leq 1$, we get

$$
\left|\Delta u_{\varepsilon}\right| \leq k t \frac{A^{k-1}}{p_{0}}\left|\nabla u_{\varepsilon}\right|+\frac{3}{2} l t \frac{A^{k}}{p_{0}}\left|\nabla u_{\varepsilon}\right|^{2}+\frac{C}{\varepsilon^{2}} .
$$

Now, let $v$ be an harmonic function such that $v=g$ on $\partial G$. Then, by applying the interpolation lemma we have

$$
\left|\nabla\left(u_{\varepsilon}-v\right)\right|^{2} \leq C\left|u_{\varepsilon}-v\right|\left|\Delta u_{\varepsilon}\right|
$$


and by $(2.14)$

$$
\left(1-C_{1} \frac{3}{2} l t \frac{A^{k}}{p_{0}}\right)\left|\nabla u_{\varepsilon}\right|^{2}-\left[k t \frac{A^{k-1}}{p_{0}}+2|\nabla v|\right]\left|\nabla u_{\varepsilon}\right|+|\nabla v|^{2}-\frac{C}{\varepsilon^{2}} \leq 0 .
$$

Direct computations show that if we choose $t$ such that $\left(1-C_{1} \frac{3}{2} l t \frac{A^{k}}{p_{0}}\right) \geq \frac{1}{2}$ we get

$$
\left|\nabla u_{\varepsilon}\right| \leq C_{2}+\sqrt{C_{3}+\frac{C_{4}}{\varepsilon^{2}}} \text { for } t \leq t_{0}=\frac{2 p_{0}}{3 l C_{1} A^{k}} \text { and } \varepsilon \leq \varepsilon_{0} .
$$

This completes the proof of $(2.8)$.

\section{$3 \quad$ Asymptotic behaviour when $\operatorname{deg}(g, \partial G)=0$}

Let us observe that if $\operatorname{deg}(g, \partial G)=0, H_{g}^{1}\left(G, S^{1}\right) \neq \emptyset$ so that the following minimum problem

$$
\min _{u \in H_{g}^{1}\left(G, S^{1}\right)} E_{\varepsilon}(u)=\min _{u \in H_{g}^{1}\left(G, S^{1}\right)} \frac{1}{2} \int_{G}\left(p_{0}+t|x|^{k}|u|^{l}\right)|\nabla u|^{2}
$$

makes sense. Our main result in this section is

Theorem 1. Let $u_{\varepsilon}$ be a minimizer of (1.1) and $u_{*}$ the unique solution of Problem (3.1). Then there exists $\bar{t}=\bar{t}\left(G, g, p_{0}, l, k\right)>0$ such that we have $\forall t \leq \bar{t}$, as $\varepsilon$ tends to 0

$$
\begin{gathered}
u_{\varepsilon} \rightarrow u_{*} \text { in } H_{g}^{1}(G, \mathbf{C}), \\
u_{\varepsilon} \rightarrow u_{*} \text { in } C^{1, \alpha}(G)
\end{gathered}
$$

and

$$
\left|u_{\varepsilon}\right| \longrightarrow 1 \text { uniformly }
$$

\subsection{Proof of Theorem 1}

In what follows, with $C$ we will denote a constant independent of $\varepsilon$. The proof of (3.2) develops into two steps.

Step 1. Proof of

$$
u_{\varepsilon} \rightarrow u_{*} \text { weakly in } H_{g}^{1}(G, \mathbf{C}) .
$$

By definition of $u_{\varepsilon}$ we have

$$
E_{\varepsilon}\left(u_{\varepsilon}\right) \leq E_{\varepsilon}\left(u_{*}\right)
$$

thus

$$
\frac{1}{2} \int_{G}\left(p_{0}+t|x|^{k}\left|u_{\varepsilon}\right|^{l}\right)\left|\nabla u_{\varepsilon}\right|^{2}+\frac{1}{4 \varepsilon^{2}} \int_{G}\left(1-\left|u_{\varepsilon}\right|^{2}\right)^{2} \leq \frac{1}{2} \int_{G}\left(p_{0}+t|x|^{k}\right)\left|\nabla u_{*}\right|^{2} \leq C .
$$


So we obtain two estimates

$$
\frac{1}{2} \int_{G}\left(p_{0}+t|x|^{k}\left|u_{\varepsilon}\right|^{l}\right)\left|\nabla u_{\varepsilon}\right|^{2} \leq C
$$

and

$$
\frac{1}{4 \varepsilon^{2}} \int_{G}\left(1-\left|u_{\varepsilon}\right|^{2}\right)^{2} \leq C
$$

Estimate (3.7) gives us

$$
\int_{G}\left|\nabla u_{\varepsilon}\right|^{2} \leq C \text { and } \int_{G}|x|^{k}\left|u_{\varepsilon}\right|^{l}\left|\nabla u_{\varepsilon}\right|^{2} \leq C
$$

which led, up to a subsequence still denoted by $\left(u_{\varepsilon}\right)$, to the following convergence

$$
u_{\varepsilon} \rightarrow u \text { weakly in } H^{1}(G, \mathbf{C}) .
$$

As $H^{1}(G, \mathbf{C}) \subset L^{4}(G, \mathbf{C})$ with compact embedding, by (3.8) we obtain

$$
\int_{G}\left(1-\left|u_{\varepsilon}\right|^{2}\right)^{2} \rightarrow \int_{G}\left(1-|u|^{2}\right)^{2}=0
$$

so $|u|=1$. Moreover, as the trace operator on $\partial G$ is continuous, we have $u_{\mathrm{\imath}} \partial G=g$ so $u \in H_{g}^{1}\left(G, S^{1}\right)$. Let us show that $u=u_{*}$.

By Lemma 2.2 we get

$$
\begin{aligned}
\int_{G}\left(p_{0}+t|x|^{k}|u|^{l}\right)|\nabla u|^{2} & \leq \underline{\lim } \int_{G}\left(p_{0}+t|x|^{k}\left|u_{\varepsilon}\right|^{l}\right)\left|\nabla u_{\varepsilon}\right|^{2} \\
& \leq \int_{G}\left(p_{0}+t|x|^{k}\right)\left|\nabla u_{*}\right|^{2}
\end{aligned}
$$

therefore $u$ is solution of Problem (3.1) and by unicity $u=u_{*}$ i.e. (3.5).

Step 2. Proof of

$$
\left\|\nabla u_{\varepsilon}\right\|_{L^{2}(G)} \rightarrow\left\|\nabla u_{*}\right\|_{L^{2}(G)}
$$

By (3.10) we have

$$
\begin{aligned}
\int_{G}\left(p_{0}+t|x|^{k}\right)\left|\nabla u_{*}\right|^{2} & \leq \underline{\lim } \int_{G}\left(p_{0}+t|x|^{k}\left|u_{\varepsilon}\right|^{l}\right)\left|\nabla u_{\varepsilon}\right|^{2} \\
& \leq \varlimsup \int_{G}\left(p_{0}+t|x|^{k}\left|u_{\varepsilon}\right|^{l}\right)\left|\nabla u_{\varepsilon}\right|^{2} \leq \int_{G}\left(p_{0}+t|x|^{k}\right)\left|\nabla u_{*}\right|^{2}
\end{aligned}
$$

and then

$$
\int_{G}\left(p_{0}+t|x|^{k}\left|u_{\varepsilon}\right|^{l}\right)\left|\nabla u_{\varepsilon}\right|^{2} \rightarrow \int_{G}\left(p_{0}+t|x|^{k}\right)\left|\nabla u_{*}\right|^{2} .
$$

Now we get

$$
\int_{G} p_{0}\left|\nabla u_{\varepsilon}\right|^{2}-\int_{G} p_{0}\left|\nabla u_{*}\right|^{2}=\int_{G}\left(p_{0}+t|x|^{k}\left|u_{\varepsilon}\right|^{l}\right)\left|\nabla u_{\varepsilon}\right|^{2}
$$




$$
-\int_{G}\left(p_{0}+t|x|^{k}\right)\left|\nabla u_{*}\right|^{2}+t \int_{G}|x|^{k}\left(\left|\nabla u_{*}\right|^{2}-\left|u_{\varepsilon}\right|^{l}\left|\nabla u_{\varepsilon}\right|^{2}\right)
$$

and then

$$
\begin{aligned}
\int_{G} p_{0}\left|\nabla u_{\varepsilon}\right|^{2} & =\int_{G}\left(p_{0}+t|x|^{k}\left|u_{\varepsilon}\right|^{l}\right)\left|\nabla u_{\varepsilon}\right|^{2}-\int_{G}\left(p_{0}+t|x|^{k}\right)\left|\nabla u_{*}\right|^{2} \\
& +\int_{G} p_{0}\left|\nabla u_{*}\right|^{2}+t \int_{G}|x|^{k}\left|\nabla u_{*}\right|^{2}-t \int_{G}|x|^{k}\left|u_{\varepsilon}\right|^{l}\left|\nabla u_{\varepsilon}\right|^{2} .
\end{aligned}
$$

If we pass to the limsup we have

$$
\begin{gathered}
\varlimsup i m \int_{G} p_{0}\left|\nabla u_{\varepsilon}\right|^{2} \leq \int_{G} p_{0}\left|\nabla u_{*}\right|^{2}+ \\
+\varlimsup \overline{\lim }\left(\int_{G}\left(p_{0}+t|x|^{k}\left|u_{\varepsilon}\right|^{l}\right)\left|\nabla u_{\varepsilon}\right|^{2}-\int_{G}\left(p_{0}+t|x|^{k}\right)\left|\nabla u_{*}\right|^{2}\right)+ \\
+t\left(\int_{G}|x|^{k}\left|\nabla u_{*}\right|^{2}-\underline{\lim } \int_{G}|x|^{k}\left|u_{\varepsilon}\right|^{l}\left|\nabla u_{\varepsilon}\right|^{2}\right) .
\end{gathered}
$$

Now, we observe that by Lemma 2.2

$$
\int_{G}|x|^{k}\left|\nabla u_{*}\right|^{2}-\underline{\lim } \int_{G}|x|^{k}\left|u_{\varepsilon}\right|^{l}\left|\nabla u_{\varepsilon}\right|^{2} \leq 0 .
$$

Then, by (3.12) and (3.13) we obtain

$$
\varlimsup \int_{G} p_{0}\left|\nabla u_{\varepsilon}\right|^{2} \leq \int_{G} p_{0}\left|\nabla u_{*}\right|^{2}
$$

which is enough to set (3.11).

Finally, (3.5), (3.11) and unicity of the limit led to (3.2) for the whole sequence.

By (2.6) and (2.8) of Proposition 2.1 we can follow [BBH2] to obtain (3.3) and (3.4).

\section{Asymptotic behaviour when $\operatorname{deg}(g, \partial G)>0$}

At first let us consider some preliminary lemmas

Lemma 4.1. Assume that $G$ is starshaped about the origin and then we have $x \cdot \nu \geq \lambda>0$ for every $x \in \partial G$. Then there is a constant $C$ depending only on $g$ and $G$ such that any solution $u_{\varepsilon}$ of Problem (2.7) satisfies

$$
\begin{gathered}
\frac{1}{\varepsilon^{2}} \int_{G}\left(1-|u|^{2}\right)^{2}+\frac{k t}{2} \int_{G}|x|^{k}|u|^{l}|\nabla u|^{2} \leq C \\
\int_{\partial G}\left|\partial_{\nu} u\right|^{2} d \sigma \leq C \\
\frac{1}{\varepsilon^{2}} \int_{G}\left(1-|u|^{2}\right)^{s} \leq C \forall s \geq 2 .
\end{gathered}
$$


Proof. As in the proof of Pohozaev identity one multiplies the equation solved by $u_{\varepsilon}$ by $x \cdot \nabla u_{\varepsilon}=x_{1} \partial_{x_{1}} u_{\varepsilon}+x_{2} \partial_{x_{2}} u_{\varepsilon}$. For clearness sake let's drop $\varepsilon$ so we obtain

$$
\begin{gathered}
\int_{G}-\operatorname{div}(p \nabla u)(x \cdot \nabla u)+\frac{l t}{2} \int_{G}|x|^{k}|u|^{l-2}|\nabla u|^{2} u(x \cdot \nabla u) \\
-\frac{1}{\varepsilon^{2}} \int_{G}\left(1-|u|^{2}\right) u(x \cdot \nabla u)=I_{1}+I_{2}+I_{3}=0 .
\end{gathered}
$$

Let us treat each term in a different way

$$
I_{1}=\int_{G}-\operatorname{div}(p \nabla u)(x \cdot \nabla u)=\int_{G} p \nabla u \nabla(x \cdot \nabla u)-\int_{\partial G} p(x \cdot \nabla u) \partial_{\nu} u d \sigma
$$

We have

$$
\nabla u \nabla(x \cdot \nabla u)=\Sigma_{i, j=1}^{2} \partial_{x_{j}} u\left(\delta_{i, j} \partial_{x_{i}} u+x_{i} \partial_{x_{i} x_{j}} u\right)
$$

then

$$
\begin{gathered}
\int_{G} p \nabla u \nabla(x \cdot \nabla u)=\int_{D} p \Sigma_{i, j=1}^{2} \partial_{x_{j}} u\left(\delta_{i, j} \partial_{x_{i}} u+x_{i} \partial_{x_{i} x_{j}} u\right) \\
=\frac{1}{2} \int_{G} p \Sigma_{i=1}^{2} x_{i} \partial_{x_{i}}|\nabla u|^{2}+\int_{G} p|\nabla u|^{2} \\
-\frac{1}{2} \int_{G} \nabla p|\nabla u|^{2} \cdot x+\frac{1}{2} \int_{\partial G} p(x \cdot \nu)|\nabla u|^{2} .
\end{gathered}
$$

Finally we have

$$
\begin{aligned}
& I_{1}=-\frac{1}{2} \int_{G} \nabla p|\nabla u|^{2} \cdot x+\frac{1}{2} \int_{\partial G} p(x \cdot \nu)\left|\partial_{\tau} g\right|^{2} \\
& -\int_{\partial G} p(x \cdot \nu)\left(\partial_{\nu} u\right)^{2} d \sigma-\int_{\partial G} p(x \cdot \tau) \partial_{\tau} u \partial_{\nu} u d \sigma .
\end{aligned}
$$

Let us consider the second term. By considering that

$$
u(x \cdot \nabla u)=\frac{1}{2} \nabla|u|^{2} \cdot x
$$

we get

$$
I_{2}=\frac{l t}{2} \int_{G}|x|^{k}|u|^{l-2}|\nabla u|^{2} u(x \cdot \nabla u)=\frac{l t}{4} \int_{G}|x|^{k}|u|^{l-2} \nabla|u|^{2}|\nabla u|^{2} \cdot x .
$$

It easy to see that

$$
I_{3}=-\frac{1}{4 \varepsilon^{2}} \int_{G}\left(1-|u|^{2}\right)^{2}
$$

By collecting together (4.7), (4.9) and (4.10) we get

$$
\begin{gathered}
-\frac{1}{2} \int_{G} \nabla p|\nabla u|^{2} \cdot x+\frac{1}{2} \int_{\partial G} p(x \cdot \nu)\left|\partial_{\tau} g\right|^{2}-\int_{\partial G} p(x \cdot \nu)\left(\partial_{\nu} u\right)^{2} d \sigma-\int_{\partial G} p(x \cdot \tau) \partial_{\tau} u \partial_{\nu} u d \sigma+ \\
\frac{l t}{4} \int_{G}|x|^{k}|u|^{l-2} \nabla|u|^{2}|\nabla u|^{2} \cdot x
\end{gathered}
$$




$$
=\frac{1}{4 \varepsilon^{2}} \int_{G}\left(1-|u|^{2}\right)^{2} .
$$

But

$$
\begin{aligned}
-\frac{1}{2} \int_{G} \nabla p|\nabla u|^{2} \cdot x & =-\frac{t}{2} \int_{G} \nabla\left(|x|^{k}|u|^{l}\right)|\nabla u|^{2} \cdot x \\
& =-\frac{t}{2} \int_{G} \nabla\left(|x|^{k}\right)|u|^{l}|\nabla u|^{2} \cdot x-\frac{1}{2} \int_{G} \nabla\left(|u|^{l}\right)|x|^{k}|\nabla u|^{2} \cdot x \\
& =-\frac{k t}{2} \int_{G}|x|^{k}|u|^{l}|\nabla u|^{2}-\frac{l t}{4} \int_{G}|x|^{k}|u|^{l-2} \nabla|u|^{2}|\nabla u|^{2} \cdot x .
\end{aligned}
$$

Finally we have

$$
\begin{gathered}
-\frac{k t}{2} \int_{G}|x|^{k}|u|^{l}|\nabla u|^{2}-\frac{l t}{4} \int_{G}|x|^{k}|u|^{l-2} \nabla|u|^{2}|\nabla u|^{2} \cdot x \\
+\frac{1}{2} \int_{\partial G} p(x \cdot \nu)\left|\partial_{\tau} g\right|^{2}-\int_{\partial G} p(x \cdot \nu)\left(\partial_{\nu} u\right)^{2} d \sigma-\int_{\partial G} p(x \cdot \tau) \partial_{\tau} u \partial_{\nu} u d \sigma+ \\
\frac{l t}{4} \int_{G}|x|^{k}|u|^{l-2} \nabla|u|^{2}|\nabla u|^{2} \cdot x=\frac{1}{4 \varepsilon^{2}} \int_{G}\left(1-|u|^{2}\right)^{2}
\end{gathered}
$$

and then

$$
\begin{gathered}
-\frac{k t}{2} \int_{G}|x|^{k}|u|^{l}|\nabla u|^{2}+\frac{1}{2} \int_{\partial G} p(x \cdot \nu)\left|\partial_{\tau} g\right|^{2} \\
-\int_{\partial G} p(x \cdot \nu)\left(\partial_{\nu} u\right)^{2} d \sigma-\int_{\partial G} p(x \cdot \tau) \partial_{\tau} u \partial_{\nu} u d \sigma=\frac{1}{4 \varepsilon^{2}} \int_{G}\left(1-|u|^{2}\right)^{2} .
\end{gathered}
$$

This directly implies (4.1) and (4.2). Now let us consider the following function

$$
h: y \in[0,1] \rightarrow \begin{cases}\frac{\left(1-y^{s}\right)}{\left(1-y^{2}\right)} & \text { if } y \neq 1 \\ \frac{s}{2} & \text { if } y=1\end{cases}
$$

It admits a maximum say $M$, so that by (4.1)

$$
\begin{gathered}
\frac{1}{\varepsilon^{2}} \int_{G}\left(1-\left|u_{\varepsilon}\right|^{s}\right)^{2} d x=\frac{1}{\varepsilon^{2}} \int_{G}\left(h\left(\left|u_{\varepsilon}\right|\right)\right)^{2}\left(1-\left|u_{\varepsilon}\right|^{2}\right)^{2} d x \leq \\
\leq M^{2} \frac{1}{\varepsilon^{2}} \int_{G}\left(1-\left|u_{\varepsilon}\right|^{2}\right)^{2} d x \leq M^{2} C .
\end{gathered}
$$

This yields (4.3).

Lemma 4.2. Let $u_{\varepsilon}$ be the solution of equation (2.7). Then there exists $t_{1}=t_{1}\left(G, g, p_{0}, l, k\right)>$ $0, \varepsilon_{0}=\varepsilon_{0}\left(G, g, p_{0}, l, k\right)>0$ such that $\forall t \leq t_{1}$

$$
\left\|\nabla u_{\varepsilon}\right\|_{4}^{2} \leq \frac{C}{\varepsilon}
$$


Proof. Using (2.7) and combining (2.13) with the fact that $\left|u_{\varepsilon}\right| \leq 1$ we obtain

$$
\left|\Delta u_{\varepsilon}\right| \leq t k \frac{A^{k-1}}{p_{0}}\left|\nabla u_{\varepsilon}\right|+\frac{3}{2} l t \frac{A^{k}}{p_{0}}\left|\nabla u_{\varepsilon}\right|^{2}+\left.\frac{1}{\varepsilon^{2}}|1-| u_{\varepsilon}\right|^{2} \mid .
$$

Then

$$
\left\|\Delta u_{\varepsilon}\right\|_{2} \leq t k \frac{A^{k-1}}{p_{0}}\left\|\nabla u_{\varepsilon}\right\|_{2}+\frac{3}{2} l t\left\|\left|\nabla u_{\varepsilon}\right|^{2}\right\|_{2}+\frac{1}{\varepsilon^{2}}\left\|1-\left|u_{\varepsilon}\right|^{2}\right\|_{2} .
$$

By (4.1) of Lemma 4.1 and by Hölder inequality

$$
\left\|\Delta u_{\varepsilon}\right\|_{2} \leq t k \frac{A^{k-1}}{p_{0}}\left\|\nabla u_{\varepsilon}\right\|_{2}+\frac{3}{2} l t\left\|\nabla u_{\varepsilon}\right\|_{4}^{2}+\frac{C}{\varepsilon} .
$$

Let us take $t \leq t_{0}$, then by (2.8) we get

$$
\left\|\Delta u_{\varepsilon}\right\|_{2} \leq \frac{3}{2} l t\left\|\nabla u_{\varepsilon}\right\|_{4}^{2}+\frac{C}{\varepsilon} .
$$

Now, let $v$ be an harmonic function with the property $v=g$ on $\partial G$. Then, by applying the Gagliardo-Nirenberg inequality we get

$$
\left\|\nabla\left(u_{\varepsilon}-v\right)\right\|_{4} \leq C_{G}\left\|u_{\varepsilon}-v\right\|_{\infty}^{\frac{1}{2}}\left\|\Delta u_{\varepsilon}\right\|_{2}^{\frac{1}{2}}
$$

and then

$$
\left\|\nabla u_{\varepsilon}-v\right\|_{4} \leq C_{G}\left(1+\|v\|_{\infty}\right)^{\frac{1}{2}}\left[C_{1}\left(\frac{3}{2} l t\right)^{\frac{1}{2}}\left\|\nabla u_{\varepsilon}\right\|_{4}+\frac{C 2}{\sqrt{\varepsilon}}\right] .
$$

Finally

$$
\left\|\nabla u_{\varepsilon}\right\|_{4} \leq\|v\|_{4}+C_{G}\left(1+\|v\|_{\infty}\right)^{\frac{1}{2}}\left[C_{1}\left(\frac{3}{2} l t\right)^{\frac{1}{2}}\left\|\nabla u_{\varepsilon}\right\|_{4}+\frac{C_{2}}{\sqrt{\varepsilon}}\right]
$$

and then

$$
\left(1-C_{3}\left(\frac{3}{2} l t\right)^{\frac{1}{2}}\right)\left\|\nabla u_{\varepsilon}\right\|_{4} \leq \frac{C_{4}}{\sqrt{\varepsilon}}+\|v\|_{4} .
$$

We choose $t$ such that $\left(1-C_{3}\left(\frac{3}{2} l t\right)^{\frac{1}{2}}\right)>\frac{1}{2}$ and this implies that

$$
\left\|\nabla u_{\varepsilon}\right\|_{4} \leq \frac{C_{5}}{\sqrt{\varepsilon}} \text { for } t<t_{1}=\min \left(t_{0}, \frac{1}{6 l C_{5}}\right) \text { and } \varepsilon \leq \varepsilon_{0}(v, C)
$$

which gives us (4.11). 


\subsection{Case $\mathrm{k}=0$}

Let us recall our energy in this case

$$
E_{\varepsilon}(u)=\frac{1}{2} \int_{G}\left(p_{0}+t|u|^{l}\right)|\nabla u|^{2}+\frac{1}{4 \varepsilon^{2}} \int_{G}\left(1-|u|^{2}\right)^{2} .
$$

By Proposition 2.1 we know that, in this case, the solution $u_{\varepsilon}$ of the minimization problem

$$
\min _{u \in H_{g}^{1}(G, \mathbf{C})} E_{\varepsilon}(u)
$$

satisfies the boundary value problem

$$
\begin{cases}-\operatorname{div}\left(p \nabla u_{\varepsilon}\right)+\frac{l t}{2}\left|u_{\varepsilon}\right|^{l-2}\left|\nabla u_{\varepsilon}\right|^{2} u_{\varepsilon}=\frac{1}{\varepsilon^{2}}\left(1-\left|u_{\varepsilon}\right|^{2}\right) u_{\varepsilon} & \text { in } G \\ u_{\varepsilon}=g & \text { on } \partial G\end{cases}
$$

Our main result here is the following one

Theorem 2. Let $\varepsilon_{n}$ a sequence going to zero and $u_{\varepsilon_{n}}$ the sequence of solutions of (4.13). Then there exist exactly $d$ points $b_{1}, b_{2}, \ldots, b_{d} \in G$ and $\bar{t}=\bar{t}\left(G, g, p_{0}, l\right)>0$ such that for every $t \leq \bar{t}$

$$
u_{\varepsilon_{n}} \rightarrow u^{*} \text { in } H_{l o c}^{1}\left(G \backslash \cup_{i=1}^{d}\left\{b_{i}\right\}\right)
$$

and

$$
E_{\varepsilon_{n}}\left(u_{\varepsilon_{n}}\right)=\pi d\left(p_{0}+t\right) \log \left(\frac{1}{\varepsilon_{n}}\right)+O(1) .
$$

The proof of this theorem needs some preliminary results.

Given $\varepsilon>0$ and $R>0$ we set

$$
I(\varepsilon, R)=\min _{u \in H_{g_{1}}^{1}}\left\{\frac{1}{2} \int_{B_{R}}\left(p_{0}+t|u|^{l}\right)|\nabla u|^{2}+\frac{1}{4 \varepsilon^{2}} \int_{B_{R}}\left(1-|u|^{2}\right)^{2}\right\}
$$

where $g_{1}(x)=\frac{x}{|x|}$ on $B_{R}$ and for $s>0$

$$
I(s)=I(s, 1) .
$$

By scaling it, it is easy to see that

$$
I(\varepsilon, R)=I\left(1, \frac{R}{\varepsilon}\right)=I\left(\frac{R}{\varepsilon}\right) .
$$


Lemma 4.3. The function $s \longmapsto\left(I(s)-2 \pi \log \frac{1}{s}\right)$ is nondecreasing, so we have

$$
I\left(s_{1}\right) \leq \pi\left(p_{0}+t\right) \log \left(\frac{s_{2}}{s_{1}}\right)+I\left(s_{2}\right) \quad \forall s_{1} \leq s_{2} .
$$

In particular

$$
I(s) \leq \pi\left(p_{0}+t\right) \log \left(\frac{1}{s}\right)+I(1) \forall s \in(0,1]
$$

Proof. Let $u_{2}$ be a minimizer for $I\left(s_{2}\right)=I\left(1, \frac{1}{s_{2}}\right)$. Set

$$
u_{1}(x)= \begin{cases}u_{2}(x) & \text { if }|x|<\frac{1}{2} \\ \frac{x}{|x|} & \text { if } \frac{1}{s_{2}}<|x|<\frac{1}{s_{1}} .\end{cases}
$$

We have

$$
\begin{aligned}
& I\left(s_{1}\right)=I\left(1, \frac{1}{s_{1}}\right) \leq \frac{1}{2} \int_{B_{\frac{1}{s_{1}}}}\left(p_{0}+t\left|u_{1}\right|^{2}\right)\left|\nabla u_{1}\right|^{2}+\frac{1}{4} \int_{B_{\frac{1}{s_{1}}}}\left(1-\left|u_{1}\right|^{2}\right)^{2}= \\
& \frac{1}{2} \int_{B_{\frac{1}{s_{2}}}}\left(p_{0}+t\left|u_{2}\right|^{2}\right)\left|\nabla u_{2}\right|^{2}+\frac{1}{4} \int_{B_{\frac{1}{s_{2}}}}\left(1-\left|u_{2}\right|^{2}\right)^{2}+\frac{1}{2} \int_{B_{\frac{1}{s_{2}}} \backslash B_{\frac{1}{s_{1}}}}\left(p_{0}+t\right)\left|\nabla \frac{x}{|x|}\right|^{2}= \\
& I\left(s_{2}\right)+\frac{1}{2} \int_{B_{\frac{1}{s_{2}}} \backslash B_{\frac{1}{s_{1}}}}\left(p_{0}+t\right) \frac{1}{|x|^{2}}=I\left(s_{2}\right)+\pi\left(p_{0}+t\right) \log \left(\frac{s_{2}}{s_{1}}\right) .
\end{aligned}
$$

By (4.18) we get

$$
I(\varepsilon, R)=I\left(\frac{\varepsilon}{R}\right) \leq \pi\left(p_{0}+t\right) \log \left(\frac{R}{\varepsilon}\right)+I(1) .
$$

Proposition 4.1. There exists $\varepsilon_{0}=\varepsilon_{0}\left(G, g, p_{0}, l, k\right)>0$ such that for $\varepsilon<\varepsilon_{0}$ and for every $t \geq 0$ we have

$$
E_{\varepsilon}\left(u_{\varepsilon}\right) \leq \pi\left(p_{0}+t\right) d \log \left(\frac{1}{\varepsilon}\right)+C
$$

where $\varepsilon_{0}$ and $C$ depend only on $g$ and $G$.

Proof. Fix $d$ distinct points $a_{1}, a_{2}, . ., a_{d}$ in $G$ and fix $R>0$ so small that

$$
\overline{B\left(a_{i}, R\right)} \subset G \forall i \text { and } \overline{B\left(a_{i}, R\right)} \cap \overline{B\left(a_{j}, R\right)}=\emptyset \forall i \neq j \text {. }
$$


Let $\Omega=G \backslash\left(\bigcup_{i=1}^{d} \overline{B\left(a_{i}, R\right)}\right)$ and consider the map $\bar{g}: \partial \Omega \rightarrow S^{1}$ defined by

$$
\bar{g}(x)= \begin{cases}g(x) & \text { if } x \in \partial G \\ e^{i \theta} & \text { if } x=a_{j}+R e^{i \theta} \in \partial B\left(a_{j}, R\right) .\end{cases}
$$

Since $\operatorname{deg}(\bar{g}, \partial \Omega)=0$, there is a smooth map $\bar{v}: \bar{\Omega} \rightarrow S^{1}$ such that $\bar{v}=\bar{g}$ on $\partial \Omega$. Then, Lemma 4.3 applied for $\varepsilon<R$ gives us

$$
E_{\varepsilon}\left(u_{\varepsilon}\right) \leq \frac{\left(p_{0}+t\right)}{2} \int_{\Omega}|\nabla \bar{v}|^{2}+\Sigma_{i=1}^{d} I(\varepsilon, R) \leq \pi\left(p_{0}+t\right) d \log \left(\frac{1}{\varepsilon}\right)+C
$$

which is the desired estimates.

Since for $t \leq t_{0}$ we have $\left\|\nabla u_{\varepsilon}\right\|_{\infty} \leq \frac{C}{\varepsilon}$ we can act as in [BBH2], Theorem III.3. Then we have the existence of $\lambda>0$ and a collection of balls $B\left(x_{i}^{\varepsilon}, \lambda \varepsilon\right), i=1, \ldots, N_{1}$ such that

$$
\left\{x \in \bar{G}:\left|u_{\varepsilon}(x)\right| \leq \frac{1}{2}\right\} \subset \bigcup_{i=1}^{N_{1}} B\left(x_{i}^{\varepsilon}, \lambda \varepsilon\right) .
$$

Given any subsequence $\varepsilon_{n}$ tending to 0 we may assume that $x_{i}^{\varepsilon_{n}}$ tend to $b_{i} \in \bar{G}$ for every $i=1, \ldots, N_{1}$. Let us denote by $\left\{b_{1}, \ldots, b_{N}\right\}$ the set of distinct $b_{i}$.

For every $j=1,2, . ., N$ we set

$$
\Lambda_{j}=\left\{i \in\left\{1,2,, . ., N_{1}\right\} ; x_{i}^{\varepsilon_{n}} \rightarrow b_{j}\right\}
$$

and

$$
d_{j}=\operatorname{deg}\left(u_{\varepsilon_{n}}, \partial B\left(b_{j}, \lambda \varepsilon_{n}\right)\right) .
$$

Fixed $\eta>0$ such that

$$
\eta<\frac{1}{2}\left|b_{i}-b_{j}\right| \forall i \neq j
$$

we consider

$$
\Omega_{j}=B\left(b_{j}, \eta\right) \backslash \bigcup_{i \in \Lambda_{j}} B\left(x_{i}^{\varepsilon_{n}}, \lambda \varepsilon_{n}\right) .
$$

Now, we are able to prove a lower bound for the functional (4.12).

Proposition 4.2. There exists a constant $C$ independent of $n, \eta$ and $\bar{t}=\bar{t}\left(G, g, p_{0}, l\right)>0$ such that, for every $j$, for every $n \geq N(\eta)$ and for every $t \leq \bar{t}$ we have

$$
\int_{\Omega_{j}}\left(p_{0}+t\left|u_{\varepsilon_{n}}\right|^{l}\right)\left|\nabla u_{\varepsilon_{n}}\right|^{2} \geq 2 \pi\left(p_{0}+t\right)\left|d_{j}\right| \log \left(\frac{\eta}{\varepsilon_{n}}\right)-C .
$$


Proof. We write on $\Omega_{j}$

$$
u_{\varepsilon_{n}}=\left|u_{\varepsilon_{n}}\right| v_{\varepsilon_{n}} \text { where } v_{\varepsilon}=\frac{u_{\varepsilon_{n}}}{\left|u_{\varepsilon_{n}}\right|} .
$$

Since $v_{\varepsilon_{n}}$ is $S^{1}$-valued and $\operatorname{deg}\left(v_{\varepsilon_{n}}, \partial B\left(x_{i}^{\varepsilon_{n}}, \lambda \varepsilon_{n}\right)\right)=d_{i}$ with $\Sigma_{i \in \Lambda_{j}} d_{i}=d_{j}$ we know that

$$
\int_{\Omega_{j}}\left|\nabla v_{\varepsilon_{n}}\right|^{2} \geq 2 \pi\left|d_{j}\right| \log \left(\frac{\eta}{\varepsilon_{n}}\right)-C
$$

(see Corollary II.1 in $[B B H 2]$ ). On the other hand, we have

$$
\left|u_{\varepsilon_{n}}\right|^{l}\left|\nabla u_{\varepsilon_{n}}\right|^{2}=\left|u_{\varepsilon_{n}}\right|^{l+2}\left|\nabla v_{\varepsilon_{n}}\right|^{2}+\left.\left|u_{\varepsilon_{n}}\right|^{l}|\nabla| u_{\varepsilon_{n}}\right|^{2}
$$

and therefore

$$
\begin{aligned}
\int_{\Omega_{j}}\left(p_{0}+t\left|u_{\varepsilon_{n}}\right|^{l}\right)\left|\nabla u_{\varepsilon_{n}}\right|^{2} & =p_{0} \int_{\Omega_{j}}\left|\nabla u_{\varepsilon_{n}}\right|^{2}+t \int_{\Omega_{j}}\left|u_{\varepsilon_{n}}\right|^{l}\left|\nabla u_{\varepsilon_{n}}\right|^{2} \\
& \geq\left(p_{0}+t\right) \int_{\Omega_{j}}\left|\nabla u_{\varepsilon_{n}}\right|^{2}-t \int_{\Omega_{j}}\left|\nabla u_{\varepsilon_{n}}\right|^{2}+t \int_{\Omega_{j}}\left|u_{\varepsilon_{n}}\right|^{l}\left|\nabla u_{\varepsilon_{n}}\right|^{2} \\
& \geq\left(p_{0}+t\right) \int_{\Omega_{j}}\left|u_{\varepsilon_{n}}\right|^{2}\left|\nabla v_{\varepsilon_{n}}\right|^{2}-t \int_{\Omega_{j}}\left(1-\left|u_{\varepsilon_{n}}\right|^{l}\right)\left|\nabla u_{\varepsilon_{n}}\right|^{2} \\
& \geq\left(p_{0}+t\right) \int_{\Omega_{j}}\left|\nabla v_{\varepsilon_{n}}\right|^{2}-\left(p_{0}+t\right) \int_{\Omega_{j}}\left(1-\left|u_{\varepsilon_{n}}\right|^{2}\right)\left|\nabla v_{\varepsilon_{n}}\right|^{2}- \\
& t \int_{\Omega_{j}}\left(1-\left|u_{\varepsilon_{n}}\right|^{l}\right)\left|\nabla u_{\varepsilon_{n}}\right|^{2} .
\end{aligned}
$$

Now we claim

$$
\int_{\Omega_{j}}\left(1-\left|u_{\varepsilon_{n}}\right|^{2}\right)\left|\nabla v_{\varepsilon_{n}}\right|^{2} \leq C
$$

and

$$
\int_{\Omega_{j}}\left(1-\left|u_{\varepsilon_{n}}\right|^{l}\right)\left|\nabla v_{\varepsilon_{n}}\right|^{2} \leq C
$$

Using the fact that $\left|u_{\varepsilon_{n}}\right| \geq \frac{1}{2}$ on $\Omega_{j}$ we see that

$$
\left|\nabla v_{\varepsilon_{n}}\right| \leq C\left|\nabla u_{\varepsilon_{n}}\right| \text { on } \Omega_{j}
$$

and therefore, by Cauchy-Schwarz,

$$
\int_{\Omega_{j}}\left(1-\left|u_{\varepsilon_{n}}\right|^{2}\right)\left|\nabla v_{\varepsilon_{n}}\right|^{2} \leq C\left\|1-\left|u_{\varepsilon_{n}}\right|^{2}\right\|_{2}\left\|\nabla u_{\varepsilon_{n}}\right\|_{4}^{2} .
$$

Let us choose $\bar{t}=t_{1}$ where $t_{1}$ is defined in Lemma 4.2. Then by (4.11), and (4.3) we have (4.24) and (4.25). Finally, we conclude using (4.23), (4.24) and (4.25). 
An argument of del Pino and Felmer see $[d P F]$ can now be used to show that (4.3) holds without the assumption on the starshapeness of $G$. In fact, applying (4.22) for $2 \varepsilon_{n}$ instead of $\varepsilon_{n}$ yields

$$
\begin{aligned}
\frac{1}{2} \int_{G}\left(p_{0}+t\left|u_{\varepsilon_{n}}\right|^{l}\right)\left|\nabla u_{\varepsilon_{n}}\right|^{2}+\frac{1}{16 \varepsilon_{n}^{2}} \int_{G}\left(1-\left|u_{\varepsilon_{n}}\right|^{2}\right)^{2} & \geq \frac{1}{2} \int_{G}\left(p_{0}+t\left|u_{2 \varepsilon_{n}}\right|^{l}\right)\left|\nabla u_{2 \varepsilon_{n}}\right|^{2}+ \\
& \frac{1}{16 \varepsilon_{n}^{2}} \int_{G}\left(1-\left|u_{2 \varepsilon_{n}}\right|^{2}\right)^{2} \\
& \geq 2 \pi d\left(p_{0}+t\right) \log \frac{1}{2 \varepsilon_{n}}-C
\end{aligned}
$$

On the other hand, by the upper bound (4.21) we have

$$
\frac{1}{2} \int_{\Omega}\left(p_{0}+t\left|u_{\varepsilon_{n}}\right|^{l}\right)\left|\nabla u_{\varepsilon_{n}}\right|^{2}+\frac{1}{4 \varepsilon_{n}^{2}} \int_{\Omega}\left(1-\left|u_{\varepsilon_{n}}\right|^{2}\right)^{2} \leq 2 \pi d\left(p_{0}+t\right) \log \frac{1}{\varepsilon_{n}}+C .
$$

Subtracting (4.27) from (4.26) yields the result. Having the estimate (4.3) on our hands, we can now follow the construction of bad-discs and complete the convergence assertion (4.15) as in [BBH2]. On the other hand, Proposition 4.1 and Proposition 4.2 give us $(4.16)$.

\subsection{Case $k>0$}

Let us recall our energy in this case

$$
E_{\varepsilon}(u)=\frac{1}{2} \int_{G}\left(p_{0}+t|x|^{k}|u|^{l}\right)|\nabla u|^{2}+\frac{1}{4 \varepsilon^{2}} \int_{G}\left(1-|u|^{2}\right)^{2}
$$

Our main result in this section is

Theorem 3. Let $\varepsilon_{n}$ a sequence going to zero and $u_{\varepsilon_{n}}$ the sequence of solutions of Problem

$$
\min _{u \in H_{g}^{1}(G, \mathbf{C})} E_{\varepsilon}(u)
$$

Then there exists $\bar{t}=\bar{t}\left(G, g, p_{0}, l, k\right)>0$ such that for every $t \leq \bar{t}$

$$
\begin{gathered}
u_{\varepsilon_{n}} \rightarrow u^{*} \text { in } H_{l o c}^{1}(G \backslash\{0\}) \\
E_{\varepsilon_{n}}\left(u_{\varepsilon_{n}}\right)=\pi p_{0}\left(d \ln \frac{1}{\varepsilon_{n}}+\frac{d^{2}-d}{k} \ln \ln \frac{1}{\varepsilon_{n}}\right)+O(1)
\end{gathered}
$$

and

$$
\operatorname{deg}\left(u^{*}, 0\right)=d
$$

At first, let us prove some preliminary results 
Proposition 4.3. There exists a subsequence $\varepsilon_{n}$ tending to 0 and a constant $C$ depending only on $g$ such that

$$
E_{\varepsilon_{n}}\left(u_{\varepsilon_{n}}\right) \leq \pi p_{0}\left(d \ln \frac{1}{\varepsilon_{n}}+\frac{d^{2}-d}{k} \ln \ln \frac{1}{\varepsilon_{n}}\right)+C
$$

Proof. Let $v_{\varepsilon}$ be a minimizer of

$$
E_{\varepsilon}(u)=\frac{1}{2} \int_{G}\left(p_{0}+|x|^{k}\right)|\nabla u|^{2}+\frac{1}{4 \varepsilon^{2}} \int_{G}\left(1-|u|^{2}\right)^{2} .
$$

Since $\left|u_{\varepsilon}\right| \leq 1$, we have $E_{\varepsilon}\left(u_{\varepsilon_{n}}\right) \leq E_{\varepsilon}\left(v_{\varepsilon}\right)$.

In the above problem, the weight function has only one point of minimum, so the thesis follows taking into account the results proved in [BH2], Theorem 1.4.

Since for $t \leq t_{0}$, we have $\left\|\nabla u_{\varepsilon}\right\|_{\infty} \leq \frac{C}{\varepsilon}$, we can act as in [BBH2], Theorem III.3. Then there exists $\lambda>0$, and a collection of balls $B\left(x_{i}^{\varepsilon}, \lambda \varepsilon\right), i=1, . ., N_{1}$ with $N_{1}$ independent of $\varepsilon$ such that

$$
\left\{x \in \bar{G}:\left|u_{\varepsilon}(x)\right| \leq \frac{1}{2}\right\} \subset \bigcup_{j=1}^{J} B\left(x_{j}^{\varepsilon}, \lambda \varepsilon\right) .
$$

Given any subsequence $\varepsilon_{n}$ tending to 0 , we may assume that $x_{i}^{\varepsilon_{n}}$ tend to $b_{i} \in \bar{G}$ for every $i=1, \ldots, N_{1}$. Let us denote by $\left\{b_{1}, \ldots, b_{N}\right\}$ the set of distinct $b_{i}$ with degree $d_{i}$.

For every $j=1,2, . ., N$ we set

$$
\Lambda_{j}=\left\{i \in\left\{1,2,, . ., N_{1}\right\} ; x_{i}^{\varepsilon_{n}} \rightarrow b_{j}\right\}
$$

and

$$
d_{j}=\operatorname{deg}\left(u_{\varepsilon_{n}}, \partial B\left(b_{j}, \lambda \varepsilon_{n}\right)\right) .
$$

Fixed $\eta>0$ such that

$$
\eta<\frac{1}{2}\left|b_{i}-b_{j}\right| \forall i \neq j
$$

we consider

$$
\Omega_{j}=B\left(b_{j}, \eta\right) \backslash \bigcup_{i \in \Lambda_{j}} B\left(x_{i}^{\varepsilon}, \lambda \varepsilon_{n}\right)
$$

Lemma 4.4. For every $j$, and $\eta<\eta_{0}$ we have $d_{j}>0$ and $b_{j}=0$ or $u_{\varepsilon_{n}}\left(b_{j}\right)=0$ for $n \geq n(\eta)$ and $t \leq t_{0}$.

Proof. For every $j$ we have

$$
\frac{1}{2} \int_{B\left(b_{j}, \eta\right)}\left(p_{0}+|x|^{k}\left|u_{\varepsilon_{n}}\right|^{l}\right)\left|\nabla u_{\varepsilon_{n}}\right|^{2} \geq \frac{1}{2} \min _{B(b j, \eta)}\left\{p_{0}+|x|^{k}\left|u_{\varepsilon_{n}}\right|^{l}\right\} \int_{B\left(b_{j}, \eta\right)}\left|\nabla u_{\varepsilon_{n}}\right|^{2} .
$$


By $[B M R]$, Theorem 3, we have for every $n \geq n(\eta)$ and $\eta<\eta_{0}$ we have

$$
\int_{B\left(b_{j}, \eta\right)}\left|\nabla u_{\varepsilon_{n}}\right|^{2} \geq \int_{\Omega_{j}}\left|\nabla u_{\varepsilon_{n}}\right|^{2} \geq 2 \pi d_{j}^{2} \ln \left(\frac{\eta}{\varepsilon_{n}}\right)-C
$$

where $C$ is a constant independent of $n$ and $\eta$. By (4.35) and (4.36) we have

$$
\frac{1}{2} \int_{B\left(b_{j}, \eta\right)}\left(p_{0}+|x|^{k}\left|u_{\varepsilon_{n}}\right|^{l}\right)\left|\nabla u_{\varepsilon_{n}}\right|^{2} \geq \min _{B(b j, \eta)}\left\{p_{0}+|x|^{k}\left|u_{\varepsilon_{n}}\right|^{l}\right\} \pi d_{j}^{2} \ln \left(\frac{\eta}{\varepsilon_{n}}\right)-C .
$$

Fix $\eta<\eta_{0}$ by (4.37) we get

$$
\frac{1}{2} \int_{G}\left(p_{0}+|x|^{k}\left|u_{\varepsilon_{n}}\right|^{l}\right)\left|\nabla u_{\varepsilon_{n}}\right|^{2} \geq \pi \ln \left(\frac{\eta}{\varepsilon_{n}}\right) \Sigma_{j} d_{j}^{2} \min _{B(b j, \eta)}\left\{p_{0}+|x|^{k}\left|u_{\varepsilon_{n}}\right|^{l}\right\}-C .
$$

Moreover by (4.33) we get

$$
\pi \ln \left(\frac{\eta}{\varepsilon_{n}}\right) \Sigma_{j} d_{j}^{2} \min _{B(b j, \eta)}\left\{p_{0}+|x|^{k}\left|u_{\varepsilon_{n}}\right|^{l}\right\}-C \leq \pi p_{0}\left(d \ln \frac{1}{\varepsilon_{n}}+\frac{d^{2}-d}{k} \ln \ln \frac{1}{\varepsilon_{n}}\right)+C
$$

As $d=\Sigma_{j} d_{j}$, in (4.39) and dividing by $\ln \frac{1}{\varepsilon_{n}}$, for $n$ large enough we obtain

$$
\Sigma_{j}\left|d_{j}\right| \leq\left(\Sigma_{j} d_{j}^{2}\right) \min _{B\left(b_{j}, \eta\right)}\left\{p_{0}+|x|^{k}\left|u_{\varepsilon_{n}}\right|^{l}\right\} \leq p_{0} \Sigma_{j} d_{j} \leq p_{0} \Sigma_{j}\left|d_{j}\right|
$$

then

$$
\Sigma_{j}\left|d_{j}\right|=\Sigma_{j} d_{j} \text { i.e. } d_{j} \geq 0 \text { for every } j .
$$

If we use (4.41) in (4.40) we get

$$
\min _{B\left(b_{j}, \eta\right)}|x|^{k}\left|u_{\varepsilon_{n}}\right|^{l} \leq 0
$$

and since $\eta$ is arbitrary (4.42) gives us $\left|b_{j}\right|^{k}\left|u_{\varepsilon_{n}}\left(b_{j}\right)\right|^{l}=0$ for every $j$ and for $n$ large enough.

The following result gives us a lower bound for the energy

Proposition 4.4. Let $u_{\varepsilon_{n}}$ be the solution of Problem (2.7). Then there exists $\bar{t}=$ $\bar{t}\left(G, g, p_{0}, l, k\right)>0$ such that for every $t \leq \bar{t}$ it holds the following estimate for the functional (4.28)

$$
E_{\varepsilon_{n}}\left(u_{\varepsilon_{n}}\right) \geq \pi p_{0}\left\{d \ln \frac{1}{\varepsilon_{n}}+\frac{d^{2}-d}{k} \ln \ln \frac{1}{\varepsilon_{n}}\right\}-C .
$$

The proof of the previous proposition needs some preliminary lemmas.

For clearness sake let us denote by $\widetilde{d}$ the degree of $u_{\varepsilon}$ around the origin. 
Lemma 4.5. Let $u_{\varepsilon_{n}}$ be the solution of Problem (2.7). Then for every $t \leq t_{1}$ it holds

$$
E_{\varepsilon_{n}}\left(u_{\varepsilon_{n}} \mid B(0, \eta)\right) \geq \pi p_{0}\left\{\widetilde{d} \ln \frac{1}{\varepsilon_{n}}+\frac{\widetilde{d}^{2}-\widetilde{d}}{k} \ln \ln \frac{1}{\varepsilon_{n}}\right\}-C
$$

where $t_{1}=t_{1}\left(G, g, p_{0}, l, k\right)$ is defined in Lemma 4.2.

Proof. We know that for $n$ large $B(0, \eta)$ contains exactly $\widetilde{d}$ bad $\operatorname{discs} B\left(x_{i}, \lambda \varepsilon_{n}\right) i \in \Lambda_{j}$ with $\left|x_{i}-x_{j}\right|>>\varepsilon_{n}^{\alpha} \forall i \neq j, \forall \alpha \in(0,1)$. Let $i_{0} \in \Lambda_{0}$ be such that $R_{n}=\max _{i \in \Lambda_{j}}\left|x_{i}\right|=\left|x_{i_{0}}\right|$. Fixing any $\alpha \in(0,1)$ we have for $n$ large enough

$$
\begin{gathered}
E_{\varepsilon_{n}}\left(u_{\varepsilon_{n}} \mid B(0, \eta)\right) \geq E_{\varepsilon_{n}}\left(u_{\varepsilon_{n}} \mid B(0, \eta) \backslash B\left(0,2 R_{n}\right)\right)+ \\
E_{\varepsilon_{n}}\left(u_{\varepsilon_{n}} \mid B\left(0,2 R_{n}\right) \backslash \bigcup_{i \in \Lambda_{0}} B\left(0, \varepsilon_{n}^{\alpha}\right)\right)+ \\
\Sigma_{i \in \Lambda_{j}} E_{\varepsilon_{n}}\left(u_{\varepsilon_{n}} \mid B\left(x_{i}, \varepsilon_{n}^{\alpha}\right) \backslash \bigcup_{i \in \Lambda_{0}} B\left(x_{i}, \lambda \varepsilon_{n}\right)\right)=E_{1}+E_{2}+E_{3} .
\end{gathered}
$$

¿From $[B M R]$ we get

$$
E_{1} \geq \pi p_{0} \widetilde{d}^{2} \log \left(\frac{\eta}{2 R_{n}}\right)-C
$$

By $[B B H 2]$ and (4.11) in Lemma 4.2 we get

$$
E_{2} \geq \pi p_{0} \widetilde{d} \log \left(\frac{2 R_{n}}{\varepsilon_{n}^{\alpha}}\right)-C
$$

for every $t \leq t_{1}$ where $t_{1}$ is defined in Lemma 4.2. Moreover

$$
\begin{aligned}
E_{3}= & \frac{1}{2} \Sigma_{\substack{i \in \Lambda_{0} \\
i \neq i_{0}}} \int_{B\left(x_{i}, \varepsilon_{n}^{\alpha}\right) \backslash B\left(x_{i}, \lambda \varepsilon_{n}\right)}\left(p_{0}+|x|^{k}\left|u_{\varepsilon_{n}}\right|^{l}\right)\left|\nabla u_{\varepsilon_{n}}\right|^{2}+ \\
& \frac{1}{2} \int_{B\left(x_{i_{0}}, \varepsilon_{n}^{\alpha}\right) \backslash B\left(x_{i 0}, \lambda \varepsilon_{n}\right)}\left(p_{0}+|x|^{k}\left|u_{\varepsilon_{n}}\right|^{l}\right)\left|\nabla u_{\varepsilon_{n}}\right|^{2} .
\end{aligned}
$$

Let us consider the last term in (4.48)

$$
\begin{gathered}
\frac{1}{2} \int_{B\left(x_{i_{0}}, \varepsilon_{n}^{\alpha}\right) \backslash B\left(x_{i 0}, \lambda \varepsilon_{n}\right)}\left(p_{0}+|x|^{k}\left|u_{\varepsilon_{n}}\right|^{l}\right)\left|\nabla u_{\varepsilon_{n}}\right|^{2}= \\
\frac{1}{2} p_{0} \int_{B\left(x_{i_{0}}, \varepsilon_{n}^{\alpha}\right) \backslash B\left(x_{i_{0}}, \lambda \varepsilon_{n}\right)}\left|\nabla u_{\varepsilon_{n}}\right|^{2}+\frac{1}{2} \int_{B\left(x_{i_{0}}, \varepsilon_{n}^{\alpha}\right) \backslash B\left(x_{i 0}, \lambda \varepsilon_{n}\right)}|x|^{k}\left|u_{\varepsilon_{n}}\right|^{l}\left|\nabla u_{\varepsilon_{n}}\right|^{2} .
\end{gathered}
$$

By $[B M R]$, as $|x| \geq\left|x_{i_{0}}\right|=R_{n}$ we can say that $|x|^{k} \geq \frac{R_{n}^{k}}{4}$ and by (4.34) we get

$$
\begin{aligned}
& \frac{1}{2} \int_{B\left(x_{i_{0}}, \varepsilon_{n}^{\alpha}\right) \backslash B\left(x_{i 0}, \lambda \varepsilon_{n}\right)}\left(p_{0}+|x|^{k}\left|u_{\varepsilon_{n}}\right|^{l}\right)\left|\nabla u_{\varepsilon_{n}}\right|^{2} \geq \\
& \pi p_{0} \ln \frac{\varepsilon_{n}^{\alpha}}{\lambda \varepsilon_{n}}+\frac{\pi R_{n}^{k}}{4}\left(\frac{1}{2}\right)^{l} \ln \frac{\varepsilon_{n}^{\alpha}}{\lambda \varepsilon_{n}}-C=\pi\left(p_{0}+\frac{R_{n}^{k}}{8}\left(\frac{1}{2}\right)^{l}\right) \ln \frac{\varepsilon_{n}^{\alpha}}{\lambda \varepsilon_{n}}-C .
\end{aligned}
$$

Finally using again $[B M R]$ and (4.49) we have

$$
E_{3} \geq \pi p_{0}(\widetilde{d}-1) \ln \frac{\varepsilon_{n}^{\alpha}}{\lambda \varepsilon_{n}}+\pi\left(p_{0}+\frac{R_{n}^{k}}{4}\left(\frac{1}{2}\right)^{l}\right) \ln \frac{\varepsilon_{n}^{\alpha}}{\lambda \varepsilon_{n}}-C
$$


Putting (4.46), (4.47) and (4.50) into (4.45) yields

$E_{\varepsilon_{n}}\left(u_{\varepsilon_{n}} \mid B(0, \eta)\right) \geq \pi p_{0}\left(\widetilde{d}^{2}-\widetilde{d}\right) \log \left(\frac{1}{R_{n}}\right)+\frac{\pi}{4}(1-\alpha) R_{n}^{k}\left(\frac{1}{2}\right)^{l} \log \left(\frac{1}{\varepsilon_{n}}\right)+\pi p_{0} \widetilde{d} \ln \frac{1}{\varepsilon_{n}}-C$.

Now let us consider the function

$$
f(s)=\pi p_{0}\left(\widetilde{d}^{2}-\widetilde{d}\right) \log \left(\frac{1}{s}\right)+\frac{\pi}{4}(1-\alpha)\left(\frac{1}{2}\right)^{l} s^{k} \log \left(\frac{1}{\varepsilon_{n}}\right)+\pi p_{0} \widetilde{d} \ln \frac{1}{\varepsilon_{n}}-C .
$$

We have

$$
f^{\prime}(s)=-\pi p_{0}\left(\widetilde{d}^{2}-\widetilde{d}\right) \frac{1}{s}+\frac{\pi}{4}\left(\frac{1}{2}\right)^{l}(1-\alpha) k s^{k-1} \log \left(\frac{1}{\varepsilon_{n}}\right) .
$$

If we impose that $f^{\prime}(s)=0$ we get

$$
s=\left(\frac{4 p_{0}\left(\widetilde{d}^{2}-\widetilde{d}\right)}{k\left(\frac{1}{2}\right)^{l}(1-\alpha) \log \frac{1}{\varepsilon_{n}}}\right)^{\frac{1}{k}} .
$$

By (4.51) and (4.52) we have

$$
\begin{gathered}
E_{\varepsilon_{n}}\left(u_{\varepsilon_{n}} \mid B(0, \eta)\right) \geq f\left(R_{n}\right) \geq f\left(\left(\frac{4 p_{0}\left(\widetilde{d}^{2}-\widetilde{d}\right)}{k\left(\frac{1}{2}\right)^{l}(1-\alpha) \log \frac{1}{\varepsilon_{n}}}\right)^{\frac{1}{k}}\right) \\
=\pi p_{0}\left\{\widetilde{d} \ln \frac{1}{\varepsilon_{n}}+\frac{\widetilde{d}^{2}-\widetilde{d}}{k} \ln \ln \frac{1}{\varepsilon_{n}}\right\}-C .
\end{gathered}
$$

Lemma 4.6. For each $j$ such that $u_{\varepsilon_{n}}\left(b_{j}\right)=0$ and for every $t \leq t_{0}$ we have, as $\varepsilon_{n} \rightarrow 0$

$$
E_{\varepsilon_{n}}\left(u_{\varepsilon_{n}} \mid B\left(b_{j}, \eta\right)\right) \geq \pi\left(p_{0}+\frac{t}{2^{l}}\left(\left|b_{j}\right|-\eta\right)^{k}\right) d_{j}^{2} \log \frac{\eta}{\lambda \varepsilon_{n}}-C .
$$

Proof. As the discs are disjoint we have $\left|u_{\varepsilon_{n}}(x)\right| \geq \frac{1}{2^{l}}$ and $|x| \geq\left|b_{j}\right|-\eta$ on $B\left(b_{j}, \eta\right) \backslash B\left(b_{j}, \lambda \varepsilon_{n}\right)$ Then we get

$$
\begin{gathered}
E_{\varepsilon_{n}}\left(u_{\varepsilon_{n}} \mid B\left(b_{j}, \eta\right)\right) \geq \frac{1}{2} \int_{B\left(b_{j}, \eta\right) \backslash B\left(b_{j}, \lambda \varepsilon_{n}\right)} p_{0}\left|\nabla u_{\varepsilon_{n}}\right|^{2}+\frac{t}{2} \int_{B\left(b_{j}, \eta\right) \backslash B\left(b_{j}, \lambda \varepsilon_{n}\right)}|x|^{k}\left|u_{\varepsilon_{n}}\right|^{l}\left|\nabla u_{\varepsilon_{n}}\right|^{2} \\
\geq \pi\left(p_{0}+\frac{t}{2^{l}}\left(\left|b_{j}\right|-\eta\right)^{k}\right) d_{j}^{2} \log \frac{\eta}{\lambda \varepsilon_{n}}-C .
\end{gathered}
$$


By Lemma 4.5 and Lemma 4.6, for every $t \leq t_{1}$ we get

$$
\begin{gathered}
E_{\varepsilon_{n}}\left(u_{\varepsilon_{n}}\right) \geq \int_{B(0, \eta)} p|\nabla u|^{2}+\int_{\bigcup_{j: b_{j} \neq 0} B\left(b_{j}, \eta\right)} p|\nabla u|^{2} \geq \pi p_{0}\left\{\widetilde{d} \ln \frac{1}{\varepsilon_{n}}+\frac{\widetilde{d}^{2}-\widetilde{d}}{k} \ln \ln \frac{1}{\varepsilon_{n}}\right\}+ \\
\pi \Sigma_{j: b_{j} \neq 0}\left(p_{0}+\frac{t}{2^{l}}\left(\left|b_{j}\right|-\eta\right)^{k}\right) d_{j}^{2} \log \frac{1}{\lambda \varepsilon_{n}}-C .
\end{gathered}
$$

Therefore as $\varepsilon_{n} \rightarrow 0$, for every $t \leq t_{1}$ it holds the following estimate for the functional $(4.28)$

$E_{\varepsilon_{n}}\left(u_{\varepsilon_{n}}\right) \geq \pi p_{0}\left\{\widetilde{d} \ln \frac{1}{\varepsilon_{n}}+\frac{\widetilde{d}^{2}-\widetilde{d}}{k} \ln \ln \frac{1}{\varepsilon_{n}}\right\}+\pi \Sigma_{j: b_{j} \neq 0}\left(p_{0}+\frac{t}{2^{l}}\left(\left|b_{j}\right|-\eta\right)^{k}\right) d_{j}^{2} \log \frac{1}{\lambda \varepsilon_{n}}-C$

where $t_{1}=t_{1}\left(\mathrm{G}, g, p_{0}, l, k\right)$ is defined in Lemma 4.2 .

Now we want to prove that the zeros of the function $u_{\varepsilon_{n}}$ are not singularities

Lemma 4.7. For $n$ large enough and for every $t \leq t_{1}$, we have $d_{j}=0 \forall j$ such that $b_{j} \neq 0$ where $t_{1}=t_{1}\left(G, g, p_{0}, l, k\right)$ is defined in Lemma 4.2.

Proof. By (4.53) and (4.33) we have

$$
\begin{gathered}
\pi p_{0}\left(d \ln \frac{1}{\varepsilon_{n}}+\frac{d^{2}-d}{k} \ln \ln \frac{1}{\varepsilon_{n}}\right)+C \geq \pi p_{0}\left\{\widetilde{d} \ln \frac{1}{\varepsilon_{n}}+\frac{\widetilde{d}^{2}-\widetilde{d}}{k} \ln \ln \frac{1}{\varepsilon_{n}}\right\} \\
+\pi \Sigma_{j}\left(p_{0}+\frac{t}{2^{l}}\left(\left|b_{j}\right|-\eta\right)^{k}\right) d_{j}^{2} \log \frac{1}{\lambda \varepsilon_{n}}-C
\end{gathered}
$$

As in (4.33) $d=\widetilde{d}$ we get

$$
\pi \Sigma_{j: b_{j} \neq 0}\left(p_{0}+\frac{t}{2^{l}}\left(\left|b_{j}\right|-\eta\right)^{k}\right) d_{j}^{2} \log \frac{1}{\lambda \varepsilon_{n}}-C \leq 0 \text { for every } j \text { and for } n \text { large }
$$

thats to say $d_{j}=0 \forall j$ such that $b_{j} \neq 0$.

Now we can complete the proof of Proposition 4.4: By Lemma 4.7 we deduce that $d=\widetilde{d}$ and if we use it in Lemma 4.5 we get the claimed lower bound (4.43). The proof of Theorem 3 is a consequence of the above results. Indeed, Proposition 4.3 and Proposition 4.4 led to (4.31) and (4.32). Finally, acting in the same way as in [BBH2] we get (4.30) with $\bar{t}=t_{1}$. Finally, the same argument of del Pino and Felmer as in (4.26) and (4.27) shows that Theorem 3 holds without the assumption of the starshapedness of $G$. 


\section{$5 \quad$ References}

[AS1] N. Andre and I. Shafrir, Minimization of the Ginzburg-Landau functional with weight, C. R. Acad. Sci. Paris Sé r. I 321(8), 999-1004, 1995.

$[A S 2]$ N. Andre and I. Shafrir, Asymptotic behaviour of minimizers for the GinzburgLandau functional with weight, Parts I and II, Arch. Rat. Mech. and Anal., 142, 1, 45-73 and 75-98, 1998.

$[B]$ G. Buttazzo, Semicontinuity, relaxation and integral representation in the calculus of variations, Pitman Research Notes in Mathematics Series, 207. Longman Scientific and Technical, Harlow; copublished in the United States with John Wiley and Sons, Inc., New York, 1989.

[BBH1] F. Bethuel, H. Brezis and F. Hélein, Asymptotic for the minimization of a Ginzburg-Landau functional, Calculus of Variations and PDE 1, 123-148, 1993.

[BBH2] F. Bethuel, H. Brezis and F. Hélein, Ginzburg-Landau vortices, Birkhäuser, 1994. [BH1] A. Beaulieu and R. Hadiji, Asymptotic for minimizers of a class of Ginzburg-Landau equation with weight, en collaboration avec A.Beaulieu, C.R. Acad. Sci. Paris, Sér. I Math., 320, no.2, 181-186, 1995.

$[B H 2]$ A. Beaulieu and R. Hadiji, A Ginzburg-Landau problem having minima on the boundary, Pro. Roy. Edinburgh Soc. A 128, 123-148, 1998.

$[B H 3]$ A. Beaulieu and R. Hadiji, Asymptotic behaviour of minimizers of a GinzburgLandau equation with weight near their zeroes, Asymptotic Analysis 22, 303-347, 2000.

$[B M R]$ H. Brezis, F. Merle and T. Rivière, Quantization effects for $-\Delta u=u\left(1-|u|^{2}\right)$ in $\mathbf{R}^{2}$, Arch. Rat. Mech. Anal. 126, 35-58, 1994.

$[D e G]$ P.G. DeGennes, Superconductivity of Metals and Alloys, Benjamin, New York and Amsterdam, 1996.

$[d P F]$ M. del Pino and P. Felmer, Local minimizers for the Ginzburg-Landau energy, Math. Z. 225, 671-684, 1997.

$[D G]$ Q. Du and M. Gunzburger, A model for supraconducting thin lms having variable thickness, Physica. D, 69, 215-231, 1994.

$[K M]$ J. Kristensen and G. Mingione, The singular set of minima of integral functionals, Arch. Rat. Mech. Anal. 180, 331-398, 2006.

$[R]$ J. Rubinstein, On the equilibrium position of Ginzburg-Landau vortices, Z angew Math Phys 46, 739-751, 1995.

$[S]$ M. Struwe, On the asymptotic behavior of minimizers of the Ginzburg-Landau model in 2 dimensions, Differential and Int. Equations 7, 1613-1624, 1994. Erratum, Differential and Int. Equations 8, 124, 1995. 